\title{
CONTROLE ROBUSTO VIA LMI PARA VEÍCULO AUTÔNOMO
}

\author{
José L. N. Silva ${ }^{1}$, Eric de Sousa ${ }^{1}$ e Anderson A. Feitosa ${ }^{1}$ \\ ${ }^{1}$ Instituto Federal de Educação e Tecnologia do Ceará (IFCE) \\ E-mail: leonardo.silva@ifce.edu.br,$\underline{\text { ericporfirio@outlook.com }}$
}

\section{RESUMO}

Neste trabalho é utilizada uma técnica de controle robusto baseada em desigualdades matriciais lineares, do inglês Linear Matrix Inequality (LMI), aplicada a um modelo de um veículo elétrico autônomo com sistema de direção elétrica. Esta estratégia de controle permite o desenvolvimento de controladores para sistemas com incertezas e otimizados por trabalhar com uma região no plano complexo, estas duas características são ideais para área automotiva onde geralmente existe incertezas dos parâmetros dos componentes utilizados e a otimização é fundamental para garantir a performance e a viabilidade econômica do produto. O objetivo do controlador é atenuar e estabilizar o veículo para entradas de distúrbios/ruídos a partir de um sistema de direção elétrica assistida, do inglês Electric Power Assisted Steering (EPAS) e também garantir o seguimento da trajetória de referência. O objetivo é a aplicação desta estratégia de controle em veículos autônomos para seguinte de trajetória de referência e rejeição a distúrbios como variações dos parâmetros do veículo, variações do meio como ventos laterais e forças de reação do solo para os pneus. O modelo matemático adotado para o estudo é obtido pela mecânica newtoniana associado a incerteza nos parâmetros dos pneus. $\mathrm{O}$ projeto do controlador conta com a implementação de restrições utilizando LMIs, cujo intuito é melhorar a resposta do sistema. As simulações realizadas visam analisar a robustez da estratégia de controle com uma restrição pelas normas H2/Hoo e desempenho pela alocação de polos definindo uma região de D-estabilidade, atendendo assim um conjunto de restrições definidas via LMIs. Os resultados de simulação obtidos apresentam as melhorias e apresentam uma nova abordagem no projeto de sistemas de controle para sistemas de controle embarcados em veículos autônomos.

\section{INTRODUÇÃO}

A definição básica de controle robusto é um ramo da engenharia de controle que lida com incertezas na representação do modelo da planta. Controladores projetados via controle robusto estão aptos a superarem pequenas diferenças entre o modelo real da planta e o modelo nominal (MAYA, 2011). Essa capacidade de lidar com incertezas no projeto de controle é muito interessante no controle de veículos autônomos visto que as incertezas nos parâmetros são constantes nos modelos matemáticos dos veículos. Na engenharia de controle clássica os controladores são projetos para um ponto de operação do sistema dinâmico a ser controlador, desta forma variações na planta afetam o desempenho e a estabilidade do controlador.

A teoria de controle robusto evoluiu ao longo das últimas décadas, apresentando soluções para vários tipos de problemas de análise, desempenho e síntese de sistemas lineares incertos. As 
desigualdades matriciais lineares (LMIs) e suas técnicas surgiram como poderosas ferramentas em diversas áreas da engenharia de controle. Uma propriedade importante das LMIs reside no fato de que o seu conjunto solução é convexo. Esta propriedade é fundamental para que se possam formular problemas em controle robusto como sendo problemas convexos de programação semidefinida, isto é, problemas de otimização convexa que minimizam uma função objetivo.

As LMIs mostram-se vantajosas na resolução de problemas que envolvem um grande número de variáveis matriciais, além de constituir um método flexível para resolver problemas relacionados ao controle de sistemas dinâmicos como veículos autônomos. Em muitos casos, o uso das LMIs pode eliminar restrições encontradas nos métodos convencionais, e ainda auxiliar na generalização de alguns tipos de problemas (SKOGESTAD, 2005). É possível posicionar os autovalores da matriz característica de um sistema em uma determinada região do plano complexo pela resolução de LMIs. O resultado é a base para o desenvolvimento de controladores robustos para a alocação de autovalores em regiões do plano complexo, regiões de D-estabilidade (AGUIRRE, 2007).

No projeto de controladores dois fatores tornam as técnicas de LMIs especiais: Uma grande variedade de especificações e restrições de projeto podem ser expressas como LMIs; Uma vez formulado em termos de LMIs, quando factível, o problema pode ser solucionado por algoritmos de otimização bastante eficientes, como o Matlab (GAHINET, 1995) e o LMISol (DE OLIVEIRA, 1997). Dentre os programas de resolução LMIs de programação semidefinida também estão o SEDUMI e o YALMIP, que se caracterizam por ser de uso iterativo e amigável, pois aproveitam do conceito de LMI adquirido pelo usuário para resolver cálculos via otimização semidefinida.

Figura 1 - Estratégia de controle com ação integral.

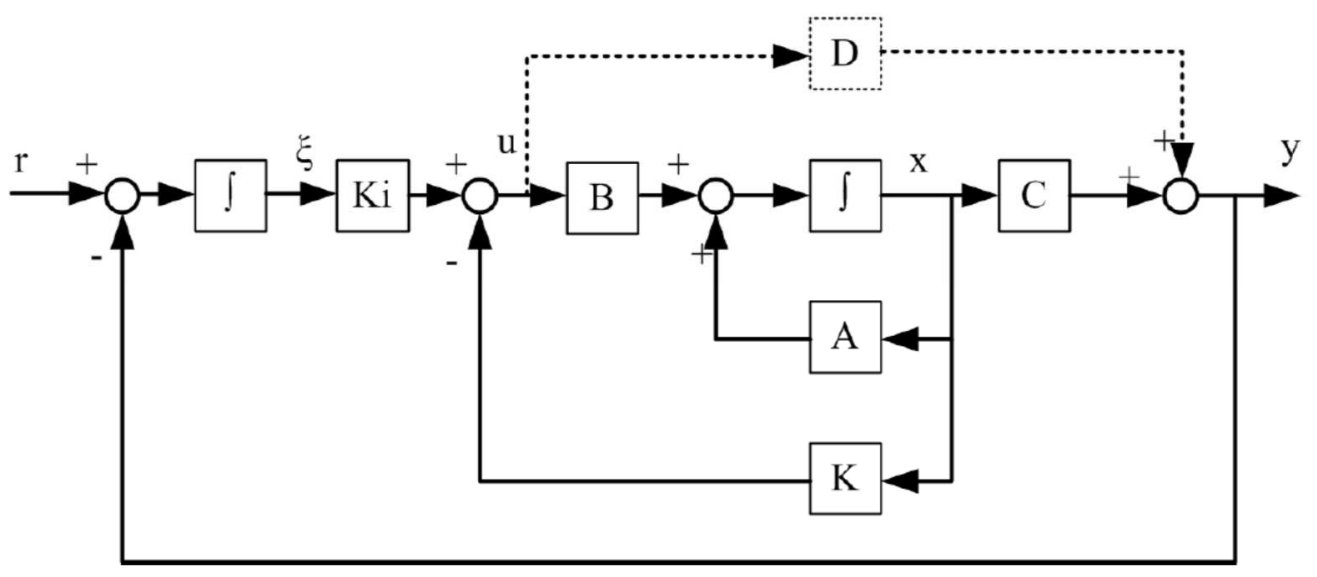

Fonte: Autores.

O conceito de LMIs é classicamente abordado em (Boyd, 1994), que é a principal referência no assunto, artigo (Chilali, 1996) propõe o uso do controle $H_{\infty}$ com uso de restrições via Destabilidade, permitindo assim a adequação de uma solução robusta aos parâmetros desejados de projeto. O trabalho (Costa, 2012) faz o uso da D-estabilidade de polos robusta numa planta multivariável, adequando o modelo robusto as condições desejadas de projeto, com este conceito não é mais necessário definir um ponto de operação. $\mathrm{O}$ controle robusto tem sido utilizado em muitas aplicações na área automotiva, controle do atuador da aceleração de motores com injeção eletrônica de combustível (ZHANG, 2015) (CORNO, 2011), controle 
integrado dos sistemas de aceleração e freios (FERGANI, 2016) (SENAME, 2013) e controle de veículos elétricos por exemplo (WANG, 2014). Em sistemas de direção com acionamento elétrico técnicas clássicas e modernas de controle são aplicadas nos seguintes trabalhos: Por exemplo em (SHAFANA, 2017) (MAROUF, 2012) para o controle da posição do EPAS é aplicada a técnica de controle denominada Sliding Mode Control. Em (CHABAAN, 2001) a técnica de controle baseada na norma Hin f é utilizada para garantir o torque de assistência no sistema EPAS. Em (SILVA, 2017) um controlador baseado em lógica fuzzy é aplicado em um sistema EPAS.

O trabalho está organizado da seguinte forma na secção 1 é proposta uma estratégia de controle robusto com ação integral via LMI no espaço de estado para o problema de seguimento de trajetória de um veículo elétrico autônomo com sistema de direção elétrica. Na secção 2 é apresentado um modelo matemático do veículo autônomo tipo SAE Fórmula E (DENTON, 2016) com incerteza politópica no parâmetro que define a aderência dos pneus ao solo. $\mathrm{Na}$ secção 3 são apresentados os resultados da aplicação da estratégia de controle proposta no modelo do veículo autônomo e em seguida a conclusão do trabalho.

\section{ESTRATÉGIA DE CONTROLE PROPOSTA}

O problema do controle de rastreamento de trajetória é geralmente definido por três aspectos gerais: o tipo de controle (lateral, longitudinal ou ambos), o tipo do modelo considerado (cinemática, dinâmica linear, dinâmicas simplificadas não lineares dinâmicas ou não lineares) e estratégia de controle a ser utilizada. Diferentes controles de rastreamento problemas foram tratados, como o controle longitudinal, o controle lateral e o misto que inclui os dois casos. $\mathrm{O}$ objetivo na tarefa de controle longitudinal é manter a velocidade linear do veículo em torno de um determinado ponto de ajuste de velocidade durante a execução com acelerações suaves. Isso é conhecido como controle de cruzeiro. Neste ponto, o motorista é liberado das tarefas de aceleração e frenagem, sendo a responsabilidade do sistema autônomo. Este caso está incluído no Nível 1 de automação definido pela SAE e um exemplo desse problema de controle é mostrado em (POLACK, 2017).

Por outro lado, o controle lateral é responsável por controlar o movimento de guinada do veículo. Para fazer isso, o controlador atua sobre o ângulo de esterçamento das rodas dianteiras. Neste caso, o condutor do veículo controla apenas a aceleração e freios, sendo o controlador automático encarregado de realizar o esterçamento. Em (FERGANI, 2016), um bom exemplo deste caso de controle é apresentado. O último controle problema é o misto. Neste caso, o controle automático realiza o movimento do veículo bidimensional completo, ou seja, o controle total das tarefas de aceleração, frenagem e esterçamento e o nível de automação sobe para os níveis 2-5, por exemplo em (ALCALA, 2018).

Para realizar o controle do seguimento de trajetória neste trabalho foi adotada a representação de sistemas dinâmico na forma de espaço de estado pelo fato de existir ferramentas matemáticas que simplificam a análise e o projeto do controlador robusto (OGATA, 2010) (DORF, 2001). O veículo autônomo deve ter seu modelo na forma de equação matricial do espaço de estado dado por:

$$
\dot{x}=A x+B u \quad x\left(t_{0}\right)=x_{0}
$$




$$
y=C x+D u
$$

Neste trabalho é proposta uma estratégia de controle robusto com ação integral via LMI para o controle de veículos autônomos. O objetivo de inserir a ação integral é garantir um erro nulo em regime permanente para o seguinte de trajetória de referência. A Figura 1 mostra o diagrama de blocos da estratégia de controle onde o bloco K e Ki são os ganhos do controlador a serem determinados via LMI.

O erro no seguimento da trajetória é dado pela seguinte equação:

$$
\xi=\mathrm{r}-\mathrm{Cx}
$$

O sinal de controle é dado pela seguinte equação:

$$
\mathrm{u}=\mathrm{K}_{\mathrm{i}} \xi-\mathrm{Kx}
$$

O sinal de controle pode ser reescrito destacando os ganhos do controlador da seguinte maneira:

$$
\mathrm{u}=-\left[\begin{array}{ll}
\mathrm{K} & -\mathrm{K}_{\mathrm{i}}
\end{array}\right]\left[\begin{array}{l}
\mathrm{X} \\
\xi
\end{array}\right]
$$

Escrevendo a estratégia de controle em malha fechada no espaço de estado tem-se:

$$
\left[\begin{array}{l}
\dot{\mathrm{x}} \\
\dot{\xi}
\end{array}\right]=\left[\begin{array}{cc}
\mathrm{A}-\mathrm{BK} & \mathrm{BK}_{\mathrm{i}} \\
-\mathrm{C} & 0
\end{array}\right]\left[\begin{array}{l}
\mathrm{x} \\
\xi
\end{array}\right]+\left[\begin{array}{l}
0 \\
\mathrm{I}
\end{array}\right] \mathrm{r}
$$

A equação (6) representada um sistema dinâmico com controlador com ação integral por realimentação de estado em malha fechada. Neste trabalho o sistema dinâmico trata de um veículo autônomo com a capacidade de seguir uma trajetória de referência. Portanto para implementar esta proposta de controle são necessários o modelo do veículo autônomo no espaço de estado e a determinação dos ganhos do controlador [K -Ki]. Este último será determinado via LMI para atender a todas as restrições adotadas para o sistema de controle.

Figura 2 - Modelo genérico de veículo.

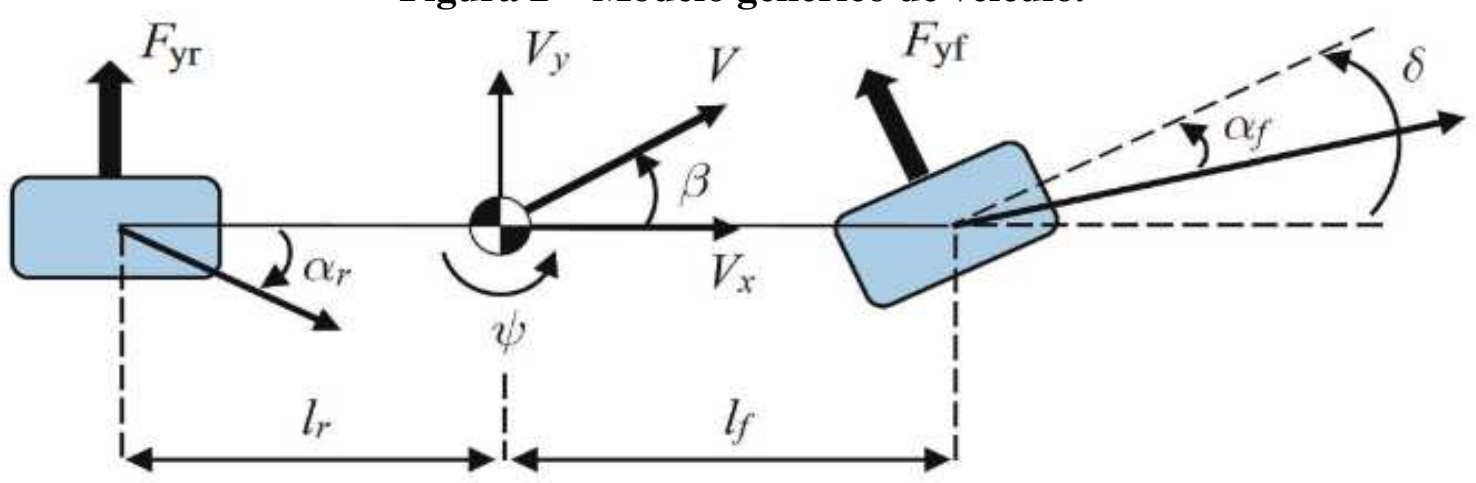

Fonte: Autores. 


\section{ESTRATÉGIA DE CONTROLE APLICADA AO MODELO}

A dinâmica do veículo elétrico autônomo tipo Formula E é aproximada pelo modelo simplificado apresentado na Figura 2. Onde Vx representa a velocidade longitudinal e Vy a velocidade transversal, neste modelo ao despreza os deslocamentos verticais define-se um controle de dois graus de liberdade. As variáveis de estado são adotadas a velocidade Vy e a velocidade angular em torno do centro de massa do veículo como descrito na equação (8).

$$
\begin{gathered}
\dot{x}=A \cdot x+B_{1} \cdot \delta+B_{2} \cdot u \\
x=\left[\begin{array}{ll}
v_{y} & \dot{\psi}
\end{array}\right]^{T}
\end{gathered}
$$

As premissas adotadas para simplificar o modelo matemático do veículo autônomo tipo Fórmula E (tração elétrica no eixo traseiro) são: as constantes de aderência dos pneus traseiros são tratadas como incertezas politópicas; a velocidade longitudinal é constante, condição aceitável por se tratar de veículo elétrico com controle digital do sistema de tração; O modelo adotado para descrever a dinâmica do veículo é o modelo de bicicleta com dois graus de liberdade representado na Figura 2 e seu modelo no espaço de estado é dado pela equação (7).

$$
A=\left[\begin{array}{cc}
-\frac{C_{\alpha f}+C_{\alpha r}}{m V_{x}} & -\left(V_{x}+\frac{l_{f} C_{\alpha f}-l_{r} C_{\alpha r}}{m V_{x}}\right) \\
-\frac{l_{f} C_{\alpha f}-l_{r} C_{\alpha r}}{I_{\mathrm{zz}} V_{x}} & -\frac{l_{f}^{2} C_{\alpha f}+l_{r}^{2} C_{\alpha r}}{I_{\mathrm{zz}} V_{x}}
\end{array}\right]
$$

Onde a matriz A representa a dinâmica do veículo descrita na equação (9), a matriz B1 representa o efeito das variações no ângulo do sistema de direção descrito na equação (10), a matriz B2 representação o sinal de controle que aciona o motor elétrico do sistema de tração descrito na equação (11).

$$
B_{1}=\left[\begin{array}{ll}
\frac{C_{\alpha f}}{m} & \frac{l_{f} C_{\alpha f}}{I_{\mathrm{zZ}}}
\end{array}\right]^{\mathrm{T}}
$$

Onde $\delta$ representa o ângulo de direção u representa o sinal de controle responsável pela manutenção da velocidade constante do veículo.

$$
B_{2}=\left[0 \frac{1}{I_{\mathrm{zZ}}}\right]^{\mathrm{T}}
$$

As forças que atuam nos pneus que representação as perturbações no sistema de direção, pois caso fossem nulas não seria necessário um controle transversal, são aproximadas pela equação (12) e seus efeitos estão incluídos na matriz A do modelo no espaço de estado.

$$
\begin{aligned}
& F_{y r}=C_{\alpha r} \cdot \alpha_{r} \\
& F_{y f}=C_{\alpha f} \cdot \alpha_{f}
\end{aligned}
$$




\section{EXEMPLO DE APLICAÇÃO EM VEÍCULO AUTÔNOMO TIPO FÓRMULA}

Como exemplo de aplicação a estratégia de controle proposta será aplicada a um veículo elétrico tipo Fórmula E autônomo. Os dados do veículo são apresentados na Tabela 1. O modelo do veículo no espaço de estado é dado pela equação (7).

Tabela 1 - Parâmetros do veículo elétrico autônomo tipo Fórmula.

\begin{tabular}{|c|c|c|}
\hline Parâmetro & Descrição & Valor \\
\hline Lr & Distância do eixo traseiro ao centro de massa & $0.717 \mathrm{~m}$ \\
\hline $\mathrm{Lf}$ & Distância do eixo dianteiro ao centro de massa & $0.873 \mathrm{~m}$ \\
\hline $\mathrm{M}$ & Massa & $356 \mathrm{Kg}$ \\
\hline $\mathrm{Ar}$ & Ângulo deslocamento lateral eixo traseiro & - \\
\hline $\mathrm{Af}$ & Ângulo deslocamento lateral eixo dianteiro & - \\
\hline $\mathrm{C} \alpha \mathrm{r}$ & Coeficiente de aderência ao solo pneu do eixo traseiro & $10 \mathrm{a} 80 \mathrm{~K} / \mathrm{rad}$ \\
\hline $\mathrm{C} \alpha \mathrm{f}$ & Coeficiente de aderência ao solo pneu do eixo dianteiro & $10 \mathrm{a} 50 \mathrm{~K} / \mathrm{rad}$ \\
\hline $\mathrm{Izz}$ & Momento de inércia & $120 \mathrm{KG} \cdot \mathrm{m}^{2}$ \\
\hline
\end{tabular}

Fonte: Autores.

Onde Car e Caf representam a incertezas politópicas associadas a constante de aderência do pneu com o solo respectivamente nos eixos traseiro e dianteiro. O modelo matemático de um sistema é uma aproximação do modelo real. Este modelo tem incertezas em seus parâmetros, que se devem ao desconhecimento dos seus valores exatos, a variações em função da temperatura, umidade etc. Estas incertezas podem ser descritas como incertezas politópicas ou limitadas em norma (AGUIRRE, 2007). Define-se um politopo de incertezas como mostrado na Figura 3.

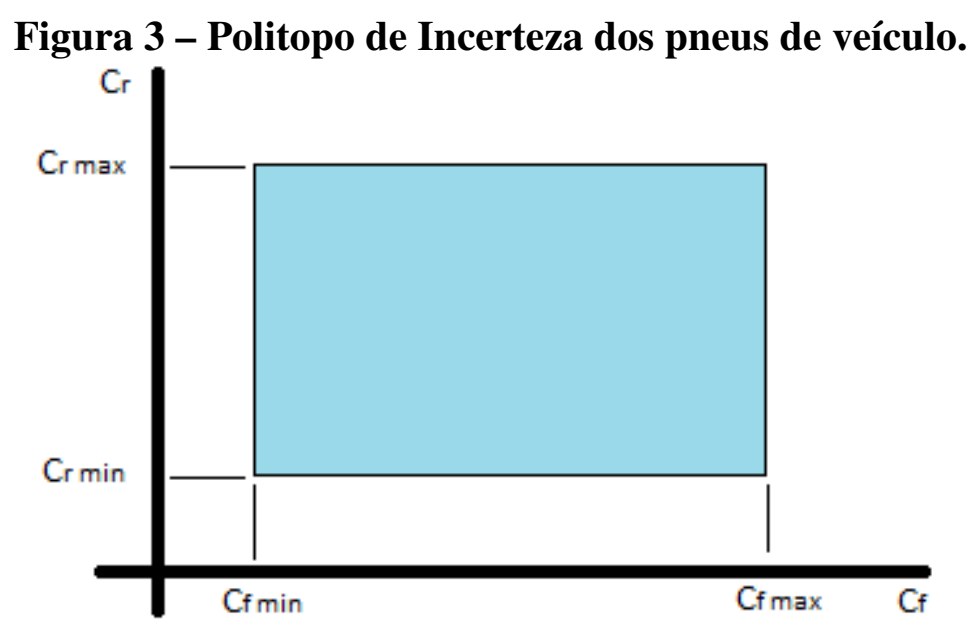

Fonte: Autores.

Na descrição das incertezas politópicas associadas aos pneus, cada parâmetro incerto é descrito como uma combinação convexa de seus valores extremos, ou seja, é possível garantir a estabilidade de um sistema incerto verificando-se a estabilidade em todos os $N$ vértices que forma a região do politopo representado na Figura 3. A Figura 4 apresenta as principais regiões de D-estabilidade adotadas para alocação de polos. 
Figura 4 - Regiões de D-estabilidade adotadas no projeto do controlador para alocação de polos via LMI.
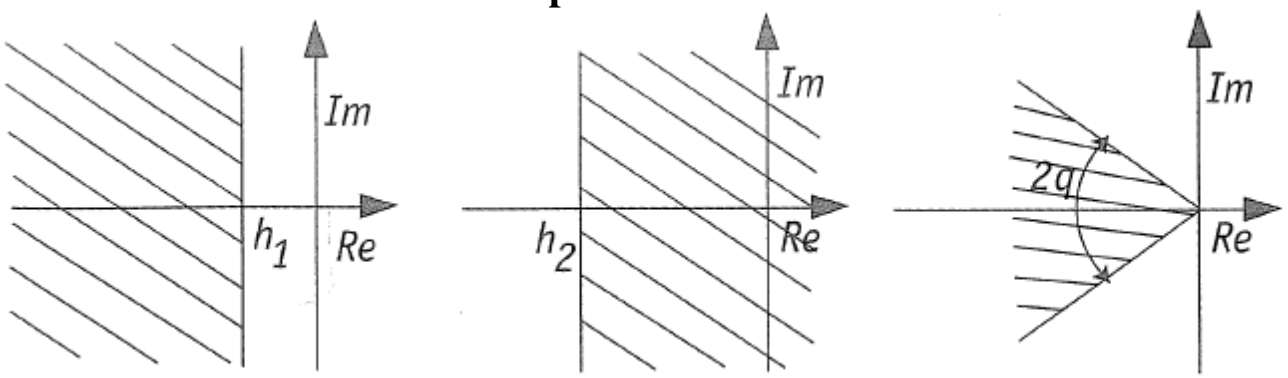

Fonte: (AGUIRRE, 2007).

A Figura 5 apresenta a região D-estabilidade resultante da adoção de uma reta vertical no ponto h1, uma reta vertical no ponto h2 e uma região cônica com ângulo 2q. Os valores de h1, h2 e q são parâmetros adotados para o projeto do controlador. Partindo da premissa que o sistema dinâmico em malha fechada deverá se comportar com um sistema de segunda ordem dado pela seguinte equação:

$$
G(s)=\frac{\omega_{n}^{2}}{s^{2}+2 \zeta \omega_{n} s+\omega_{n}^{2}}
$$

Analisando a resposta dinâmica deste sistema e adotando um valor de sobressinal máxima de $10 \%$ e um tempo acomodação máximo de 1 segundo, os parâmetros adotados foram $\mathrm{h} 1=-5$, h2=-100 e q=0.4365 rad. Os valores de sobressinal e tempo de acomodação máximos são escolhas de projeto, os valores adotados neste visam um bom desempenho em competição.

\section{Figura 5 - Alocação dos polos via LMI na região D-estabilidade definida para o sistema} em malha fechada.

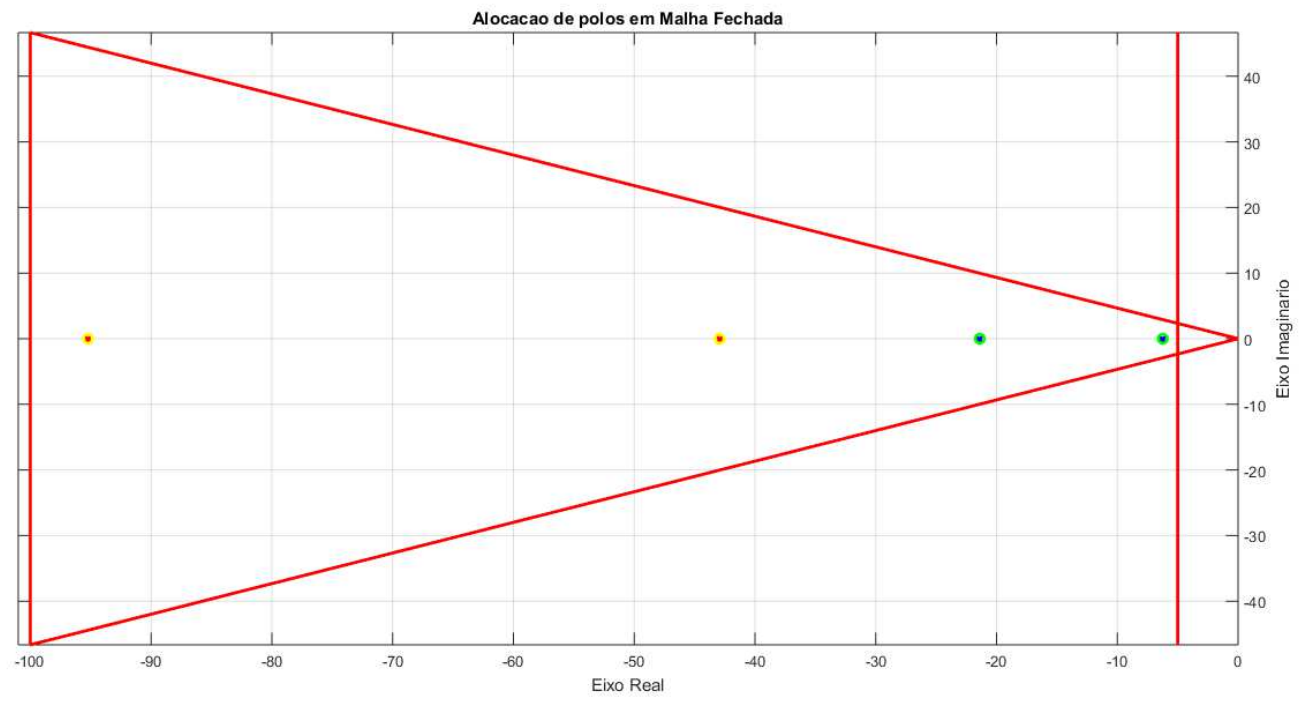

Fonte: Autores.

A Figura 6 mostra a resposta do veículo a uma entrada degrau, ou seja, é mostrado como varia a velocidade lateral $\mathrm{Vy}$ e rotação no centro do veículo $\psi$ para uma variação de $90^{\circ}$ no ângulo 
da direção. Essas respostas são para o sistema sem ação do controlador em malha aberta. É importante destacar que para cada resposta tem duas curvas em função dos limites máximo e mínimos da incerteza politópica. A variável $\mathrm{Vy}$ atingi valores finais na faixa de [0.012 a -0.055] $\mathrm{m} / \mathrm{s}$ e a variável $\psi$ atingi valores finais na faixa de [0.039 a 0.048$] \mathrm{rad} / \mathrm{s}$.

\section{Figura 6 - Resposta ao Degrau em Malha Aberta.}

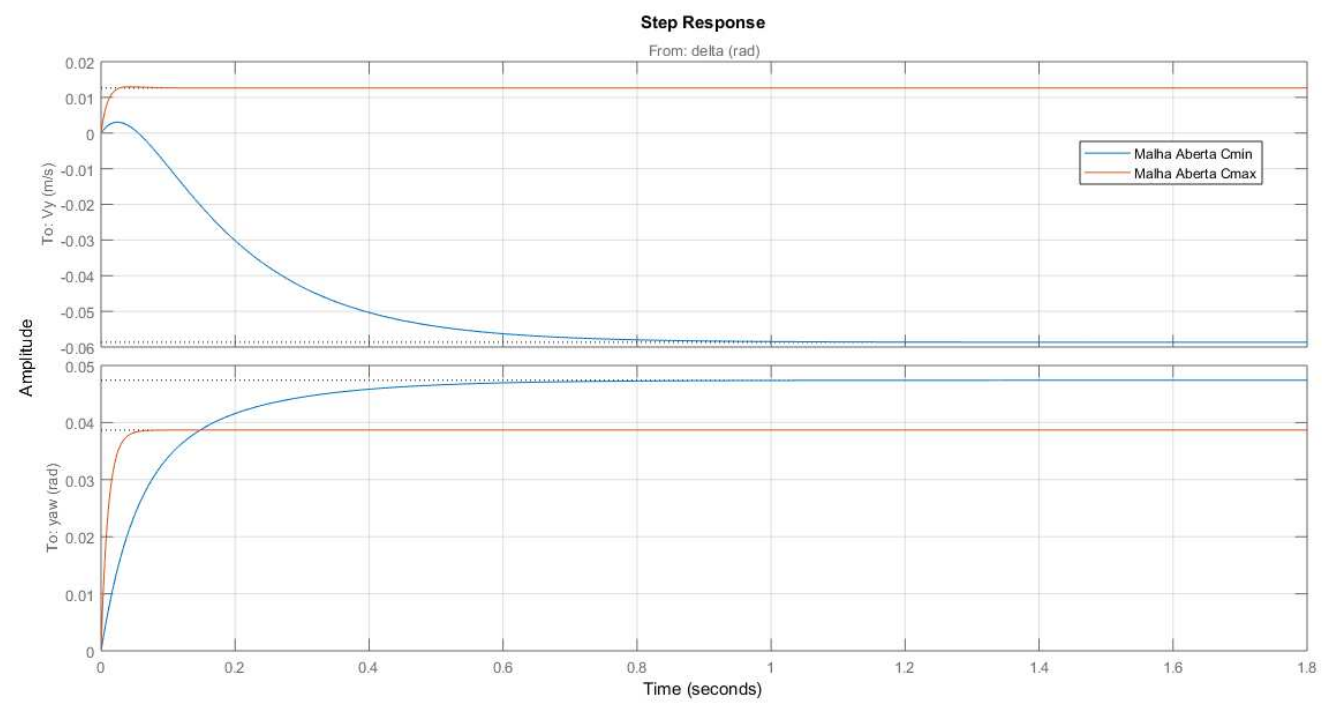

Fonte: Autores.

A Figura 7 mostra a resposta do veículo a uma entrada degrau de $90^{\circ}$ no ângulo da direção $\delta$. Desta vez com a estratégia de controle proposta, ou seja, sistema em malha fechada. É importante destacar que para cada resposta tem duas curvas em função dos limites máximo (curva azul) e mínimos (curva vermelha) da incerteza politópica nos parâmetros dos pneus. A variável Vy atingi valores finais na faixa de $[0.001$ a -0.011$] \mathrm{m} / \mathrm{s}$ e a variável $\psi$ atingi valores finais na faixa de [0.0001 a 0.0006] rad/s. Portanto, melhorias consideráveis nas respostas.

Figura 7 - Resposta ao Degrau em Malha Fechada.

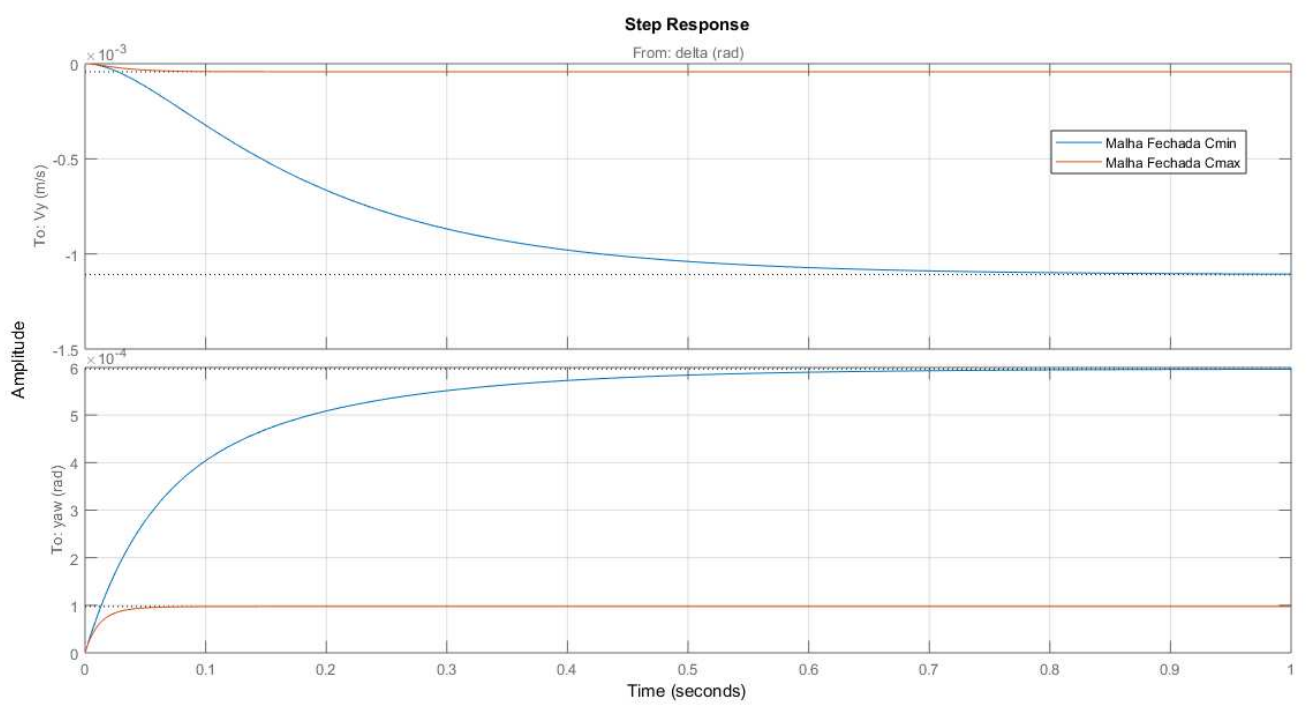

Fonte: Autores. 
É possível observar uma melhora também na robustez do sistema a distúrbios/ruídos com o uso das normas $\mathrm{H} 2$ e Hinf. A Figura 8 apresenta a resposta do sistema de controle proposto no domínio da frequência. A curva na cor vermelha representa o sistema em malha aberta, a curva na cor azul mostra que a energia dos distúrbios/ruídos é atenuada desta forma garantindo uma robustez da estratégia de controle proposta. A figura apresenta uma curva para cada variável controlada Vy e $\psi$.

Figura 8 - Análise da Robustez no Domínio da Frequência.
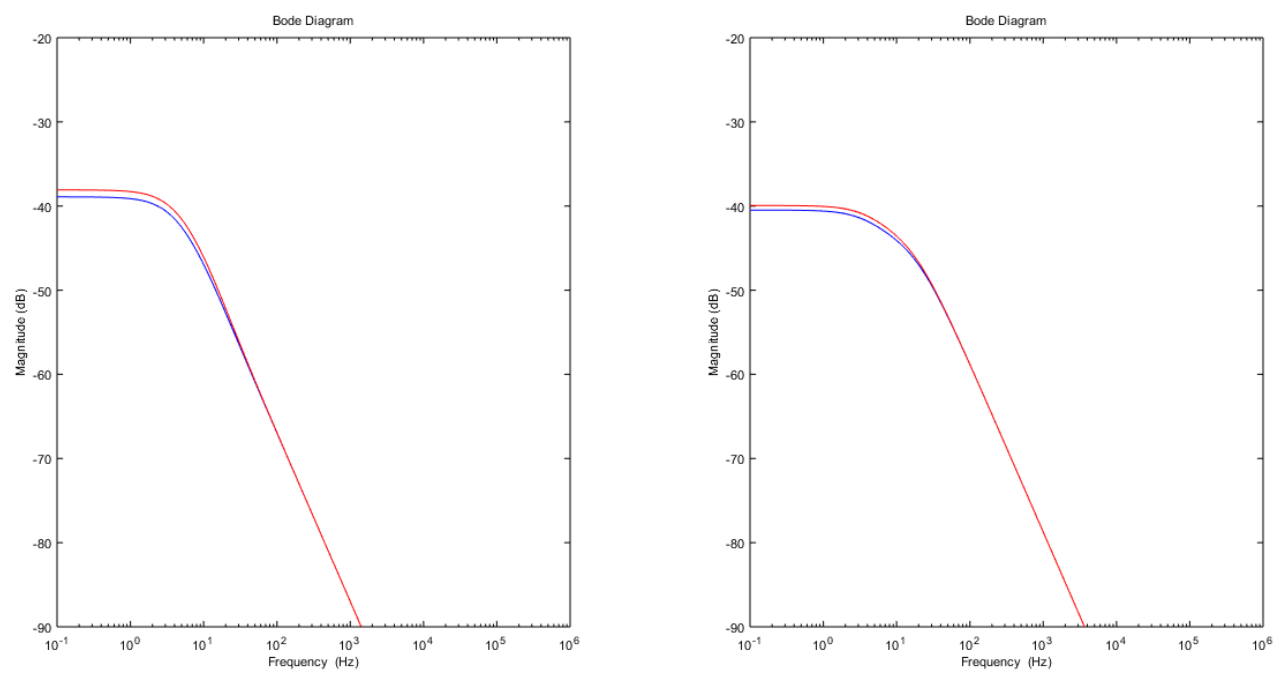

Fonte: Autores.

\section{CONCLUSÃO}

Neste trabalho foi proposta uma estratégia de controle robusto para o controle seguimento de trajetória de um veículo autônomo. O desempenho do controlador se mostrou satisfatório nas simulações computacionais assim como sua capacidade de atenuar perturbações. Outro ponto interessante é a aplicação da incerteza do modelo da dinâmica veículo. O trabalho aplicou técnicas de controle que os autores entendem serem mais adequadas para o controle de veículos terrestres em função das grandes variações e incertezas associadas aos seus componentes. Esta pesquisa é uma contribuição para o desenvolvimento dos veículos autônomos e está numa fase inicial os próximos passos são a implementação deste controlador em um veículo tipo Fórmula E e em seguida sua integração com outros controladores embarcados como sistemas de frenagem, sistema de tração e sistema de navegação.

\section{REFERÊNCIAS}

AGUIRRE, L. A. Enciclopédia de Automática: Controle e Automação, vol. 1. São PauloSP. Blucher, 2007.

ALCALA, E.; PUIG, V.. Autonomous vehicle control using a kinematic lyapunov-based technique with lqr-Imi tuning. Control Engineering Practice, Elsevier, v. 73, p. 1-12, 2018. 
BOYD, S., GHAOUI, L., FERON, E., BALAKRISHNAN, V. Linear Matrix Inequalities in Systems and Control Theory. Philadelphia, 1994.

DENTON, TOM. Electric and Hybrid Vehicles. Routledge, 2016.

DORF, R.; BISHOP, R. Sistemas de controle modernos. Rio de Janeiro: LTC Editora, 2001.

CORNO, M.; TANELLI, M.; SAVARESI, S. M.. Design and validation of a gain-scheduled controller for the electronic throttle body in ride-by-wire Racing motorcycles. IEEE Transactions on Control Systems Technology, IEEE, v. 19, n. 1, p. 18-30, 2011.

CHABAAN, R. C.; WANG, L. Y. Control of electrical power assist systems: $\mathbf{H}$ inf design, torque estimation and structural stability. JSAE review, Elsevier, v. 22, n. 4, p. 435-444, 2001.

MAROUF, A.; DJEMAI, M.; SENTOUH, C.; PUDLO, P. A new control strategy of na electric-power-assisted steering system. IEEE Transactions on Vehicular Technology, IEEE, v. 61, n. 8 , p. 3574-3589, 2012.

MAYA, Paulo A.; LEONARDI, Fabrizio. Controle essencial. Pearson Prentice Hall, 2011.

OGATA, K. Engenharia de Controle Moderno. 4 ed. Pearson Brasil, 2010.

POLACK, P.; D'ANDRÉA-NOVEL, B.; FLIESS, M.; FORTELLE, A. D. L.. Finite-time stabilization of longitudinal control for autonomous vehicles via a model-free approach. IFAC-PapersOnLine, Elsevier, v. 50, n. 1, p. 12533-12538, 2017.

SKOGESTAD S.; POSTLEHWAITE, I. Multivariable Feedback Control: Analysis and Design. 2 ed. Wiley, 2005.

SILVA, JOSÉ L. N. Sistema de Direção Elétrica Assistida com Inteligência Artificial. XXV Simpósio Internacional de Engenharia Automotiva - SIMEA, São Paulo: Blucher, p. 737-751, 2017.

SENAME, O.; GASPAR, P.; BOKOR, J. Robust control and linear parameter varying approaches: application to vehicle dynamics. [S.1.]: Springer, 2013. v. 437.

SHAFANA, P.; MUHAMMED, S. Admittance based position tracking using sliding mode controller for an electric power steering system. In: IEEE. Intelligent Computing and Control Systems (ICICCS), 2017 International Conference on. [S.1.], 2017. p. 574-578.

WANG, R.; ZHANG, H.; WANG, J. Linear parameter-varying controller design for fourwheel independently actuated electric ground vehicles with active steering systems. IEEE Transactions on Control Systems Technology, IEEE, v. 22, n. 4, p. 1281-1296, 2014.

ZHANG, S.; Lpv modeling and mixed constrained $h 2=h i n$ f control of an electronic throttle. IEEE/ASME Transactions on Mechatronics, IEEE, v. 20, n. 5, p. 2120-2132, 2015. 